\title{
Cellular receptor binding and entry of human papillomavirus
}

Tan Letian ${ }^{1}$, Zhang Tianyu ${ }^{2^{*}}$

\begin{abstract}
Human papillomaviruses (HPVs), recognized as the etiological agents for the skin, plantar, genital, and laryngopharyngeal wart, have been previously in numerous studies demonstrated to present a close link between HPV infection and certain human cancers, some putative candidates of HPV cell receptor and possible pathways of cell entry proposed. This review was to highlight the investigations and remaining questions regarding the binding and entry process.
\end{abstract}

\section{Introduction}

As the well-recognized etiological agents for the skin, plantar, genital, and laryngopharyngeal wart, human papillomaviruses (HPVs) have been proven of a close link between HPV infection and certain human cancers. And considerable effort has been made in developing a prophylactic vaccine and devising effective treatments of HPV-induced lesions. It has been found that the early events of HPV infection such as cellular receptor binding and entry into susceptible cells could provide potential targets of inhibiting the spread of a HPV infection.

HPVs are nonenveloped double-strained DNA viruses about $55 \mathrm{~nm}$ in diameter with an approximately 8-kb genome in the nucleohistone core, and their capsids are composed of two virally encoded proteins, L1 and L2, with L1, the major capsid protein, mainly responsible for initial binding to the cell surface, and arranged in 72 pentamers which associate with $\mathrm{T}=7$ icosahedral symmetry [1]. Even in the absence of other viral proteins, L1 self-assembles into empty capsid or virus-like particles (VLPs) [2]; L2 is incorporated into VLPs when coexpressed with L1, i.e. L1/L2 VLPs, in insect or mammalian cells [1]. It is well known that difficulties in generating HPVs in vitro hinder the study on the pathway of infection. Recent research predominantly utilized synthetic HPV particles, such as VLPs, HPV-based gene transfer vectors known as pseudovirions (PsV), or papillomavirus genome-containing quasivirions (QV). VLPs, including L1 VLPs or L1/L2 VLPs, are widely used for

\footnotetext{
* Correspondence: ty.zhang2006@yahoo.com.cn

${ }^{2}$ Department of Otolaryngology, Eye and ENT Hospital, Fudan University, Shanghai, China
}

the binding assay and vaccine production. PsV, produced for the studies on internalization, are composed of the VLPs packaging or attached to a reporter gene whose subsequent expression is used to identify and quantity pseudoinfected cells [3]; QV are generated as in the case of PsV in that the transfection of L1 and L2 codon-optimized expression plasmids, in addition to full-length, recircularized HPV genomes, into 293T or 293TT cells allows for efficient intracellular production of the native virion-like particles [4].

However, native virions are generated in stratified and differentiated epithelia and are thus synthesized only during a natural infection or in an organotypic culture [4]. For years, the low productivity of organotypic culture garnered too few virions for many low sensitivity analyses. Recently, Broker's group established a highly efficient and reproducible system that generated autonomous HPV-18 genome in the primary human keratinocytes, the organotypic raft cultures of which recapitulated a robust productive program, which suggested potential value for HPV genetic dissection and a faithful ex vivo model for investigating infections and interventions [5,6].

It is well known that HPVs, prior to a successfully established infection, have to experience a complicated process to bind to and enter the host cell. Our review was intended as an update on the cellular receptor and endocytic route of HPVs, with a focus on each putative receptor and a possible pathway based on the previous evidence derived from the literature review. In addition, we briefly clarified the function of L2 protein in HPV infection. 


\section{HPV receptor binding and virus-host cell interaction}

The identification of HPVs cellular receptor began with the observation of interaction between papillomaviruses and cells. Roden et al observed that BPV-1 virions, as well as VLPs of HPV-16, were capable of binding specifically to several mammalian cell lines of fibroblastic and epithelial origin [1]. Afterwards, HPV-11, -16 and -33 VLPs were reported to have bound to or entered a wide range of cells $[7,8]$. These findings suggested that papillomavirus receptor was a widely expressed and evolutionally conserved surface receptor. Later, Qi et al reconfirmed the outcomes, and, furthermore, proved the receptor as a trypsin-sensitive structure and identified a B-cell line DG-75 that did not bind VLPs, which was critical to the investigations to come [9].

\section{$1.1 \alpha 6$ integrin}

The first candidate of cellular receptor was nominated as $\alpha 6$ integrin $[2,10,11]$. The integrins, heterodimeric glycoproteins comprising of $\alpha$ and $\beta$ subunits, are expressed in a variety of cell types, primarily involved in cell-matrix and cell-cell interactions, and as the previous researches revealed, capable of acting as virus receptors for initial binding and/or internalization, as in the case of echovirus ( $\alpha 2 \beta 1)$, coxsackievirus ( $\alpha v \beta 3)$, hantavirus $(\beta 3)$, adenovirus $(\alpha v \beta 3 / 5)$, and foot and mouth disease virus $(\alpha v \beta 3)[2]$.

At present, 17 types of $\alpha$ subunits and eight of $\beta$ subunits are known, of which $\alpha 6, \beta 4$, and $\beta 1$ have been reported to be involved in HPV binding. And it has been clarified that $\alpha 6$ integrin played a leading role in this process with the evidence that a monoclonal antibody against the $\alpha 6$ integrin subunit reduced the binding degree of VLPs, while anti- $\beta 1$ or anti- $\beta 4$ antibodies did not [2]; that HPV6 bL1 VLPs failed to bind to DG75 [2], whereas VLPs did to the genetically modified DG-75 expressing $\alpha 6$ integrin [10]; that the binding degree of HPV-16 VLPs to each cell type varied not with the expression level of $\beta$ subunits, but with that of the $\alpha 6$ integrin [11]. In addition, the $\alpha 6$ integrin was known capable of invoking a transductive signal pathway to initiate DNA replication in keratinocytes, which could be an ideal condition for viral replication [10].

Although both the $\alpha 6 \beta 4$ and $\alpha 6 \beta 1$ heterodimer were capable of binding VLPs in vitro, only the former was supposed to function as a HPV receptor, which was concluded from the results that the $\alpha 6$ subunit associated preferentially with the $\beta 4$ in epithelial cells [12], and the $\alpha 6 \beta 4$ complex was expressed exclusively in the basal cellular layer of the stratified squamous epithelium [13], which was presumably the only site of productive PV infection, while the $\alpha 6 \beta 1$ was found in relatively more cell types and sites unrelated to HPV infection, which might partly explain why HPVs were reported to be able to bind to a wide range of cells in vitro that were not the natural host of HPVs [10]. And the consistent results from Evander et al showed that the human laminin, the natural ligand for the $\alpha 6 \beta 4$ complex, was capable of blocking the binding of VLPs to HaCaT cells in a dose-dependent manner [2].

It is generally held that HPV infection is believed to occur as a result of exposure of basal cells to virus particles upon a minor trauma to the epithelium. During the wound healing, the $\alpha 6 \beta 4$ complex presented a high expression over the entire surface of the epithelial cells migrating to cover the focus [14]. Furthermore, the complex was constitutively endocytosed and recycled, with a rate of endocytosis of $1 \%$ to $2 \%$ of surface molecules per min, and with a recycle of the receptor facilitating the cellular migration, during which it moved in and out as the cell was advancing [15]. Therefore, a model of HPV infection was proposed that HPV particles bound to the $\alpha 6 \beta 4$ complex during the wound healing and were endocytosed to the microfilament network via the hemidesmosome [2], of which the complex is an integral part [16].

\subsection{Heparan sulfate proteoglycans/Heparan sulfate 1.2.1 Heparan sulfate proteoglycans/Heparan sulfate and HPV binding}

Cell surface heparan sulfate proteoglycans (HSPGs), mainly syndecans and glypicans, are complex molecules composed of a core protein with covalently attached glycosaminoglycans chains, especially heparan sulfate. The glycosaminoglycans, comprised of alternating disaccharide units of uronic acid and amino sugars, are posttranslationally modified by sulfation and acetylation to various degrees, providing a variety of molecules with substantial sequence heterogeneity [17]. HSPGs are involved in a wide variety of biological phenomena, including organogenesis, angiogenesis, growth factors/ cytokine actions, wound healing, and cell adhesion. Moreover, they are implicated as primary host cell receptors for many viruses, although most of them depend on secondary receptor proteins for efficient internalization [18].

As part of HSPGs, heparan sulfate was reported to play a critical role in the binding of HPVs to the cell surface: 1) the removal of heparan sulfate glycosaminoglycans on keratinocytes with heparinase or heparitinase resulted in an $80 \%-90 \%$ reduction of HPV-11 VLPs binding [19]; 2) the pseudoinfection of HPV-16 and -33 was inhibited by heparin, reduced with a decline in the level of surface sulfation, and abolished via a heparinase treatment [20]; 3) HPV-16, -18, -31, -33, -39, -45, -58, -59 , and -68 VLPs possessed the ability to transfer genes into COS-7 cells in an efficient way, which, however, 
was inhibited when the pseudovirions were preincubated with heparin [21].

Of HSPGs, syndecan-1, instead of syndecan-4 and glypican-1, was reported to function as a HPV receptor. Evidence were: 1) when cells was treated with heparinase I, rather than with phosphoinositol-specific phospholipase $C$, which could remove most of the surface heparan sulfate, the degree both in their binding of VLPs and infection with HPV-33 pseudovirions was sharply reduced [22]; 2) K562 cell with ectopic expression of syndecan-1 could enhance its binding of HPV-16 VLPs, which otherwise possessed no HSPGs but minor amounts of molecules and thus weakly bound of VLPs [23]. Syndecan-1 was strongly upregulated during the wound healing, and widely expressed on the migrating and proliferating keratinocytes as well as on the adjacent hair follicles. Therefore, the basal keratinocytes, in addition to the suprabasal ones, when exposed upon a minor trauma or abrasion, overexpressed syndecan-1, thus upregulating strongly their ability to bind and internalize papillomaviruses in vivo [23].

\subsubsection{Conformation of HPV virions and HPV binding}

The conformation of HPV particles is considered to be critical to the cellular binding. Initially, Joyce et al identified a conserved heparan-binding region on the carboxyl-terminal portion of HPV L1 protein through a sequence comparison of nine HPV types: HPV-11, -3 , $-13,-31,-58,-6 \mathrm{~b},-40,-7$ and -42 . This region was found to be located in the final 15 amino acid residues of the L1 protein of the general type XBBBBXB where $\mathrm{B}$ was Lys, Arg or His, which was similar to the XBBXBX and XBBBXXBX consensus sequences of the known heparin-binding proteins [19]. However, later studies reported that deletion of this region did not affect the interaction of HPV-33 VLPs with heparin [20], and the interaction was strictly dependent on an intact outer surface conformation of L1 [24], suggesting that the basic C-terminus of $\mathrm{L} 1$ was not sufficient for heparin binding. Moreover, the structure of the papillomavirus capsid, reported recently, showed that the C-terminus was not surface-exposed [25,26]. Taken together, these data indicated that the interaction between the capsid and heparin required an intact outer surface structure, which provided a conformational cluster of basic amino acids rather than a linear arrangement of positively charged amino acids [24].

Afterwards, Selinka et al, based on published and their own data, proposed that papillomavirus virions might exist in two conformational forms, the closed and open, the former the predominant species in solution, from which the binding of the surface receptors cause a transition in the virion to the latter, which might initiate internalization and uncoating [17]. Day et al later on demonstrated this model in details via a study on neutralization of HPV with monoclonal antibodies [27]. In the study, three anti-HPV-16 monoclonal antibodies were employed, which were H16.V5 (V5), H16. E70 (E70), and H16.U4 (U4). And the result showed that V5 and E70, recognizing overlapping epitopes present on the apex of the L1 capsomers [28], did not interfere with the virion binding to the cell surface, but neutralized infection by preventing the internalization of bound particles [27]; that U4, whose epitope was mapped to a C-terminus portion of $\mathrm{L} 1$, and was proposed to extend between adjacent capsomers [28], interfered with infection by preventing cellular binding, but did not interfere with the binding to ECM. These data suggested that interaction between HPV and cells was dependent on functional epitopes on the particle [27]. As in the case of the U4 epitope, it physically overlapped with a HSPGs binding site within cleft within which there might be a heparin-binding domain, which, however, might not be the site originally proposed by Joyce et al, and cell-induced conformational changes could expose the C-terminus of L1, resulting in a higher affinity binding between virions and cells. On the other hand, several epitopes, such as V5 and E70, once occupied, could prevent a necessary conformational change in capsid, or might induce a conformational change in another way, in order to block the binding [27].

\subsubsection{Structure of HSPGs and HPV binding}

The structure of HSPGs is believed to be equally essential. For instance, O sulfation of HSPGs was sufficient for VLPs binding, which, however, was required together with $\mathrm{N}$ sulfation by pseudoviruses [17]. This, nevertheless, has received little research. So far, it has been recognized that 2-O-sulfate groups, primarily located on iduronic acid residues in heparin and heparan sulfate, glucosmine $\mathrm{N}$-sulfate and in particular glucosamine 6$\mathrm{O}$-sulfate groups of the polysaccharide all contribute to the interaction with HPV-16 VLPs. In addition, eight monosaccharide units of heparin were sufficient for the binding of HPV-16 VLPs, which increased as the heparin chain prolonged in size from eight to 14 units, but decreased with 16 or more units [18].

It still remains mysterious whether $\alpha 6$ integrin or HSPGs is the genuine cellular receptor of HPV, for there are counterevidences and controversies for either. Several studies indicated that $\alpha 6$ integrin was dispensable for HPV-11 VLP binding to cells [19], for BPV-4 infection [29], as well as for HPV-16 and HPV33 pseudoinfection [20]. On the other hand, HSPGs, especially heparan sulfate, was not required for HPV31b virions infection of human keratinocytes in vitro [3]. However, Johnson et al recently showed the opposite results using the murine cervicovaginal challenge model that in vivo HPV-31 infection was dependent on HSPGs [30]. We put forwarded at least three 
possible explanations to the discrepancies between the outcomes. In the first place, it was largely that the different assay systems were employed since a standardized one was not available, where the viral particles could be VLPs, pseudovirions, or authentic virions; and the cell lines, diversified, including those derived from malignant carcinomas, such as Hela and $\mathrm{HaCaT}$, etc, and the normal keratinocytes from human beings or animals. Different kinds of viral particles required different concentrations in assay. For example, MOIs in the setting of authentic virions ranged from 5 to 50 viral genome equivalents per cell, but reached thousands to tens of thousands per cell in most cases of VLP binding or pseudovirion pseudoinfection [3]. And different cell lines presented distinct characteristics. Those transformed cells, which lost some of their epithelial characteristics, might result in disparities. Second, HPVs of different types employed distinct molecules as their own primary receptors. This could be the simplest explanation, which needs further evidence. In addition, further studies suggested that HPV infection was likely to engage more than one cellular surface protein, as in the case of a secondary receptor $[10,19,20,23]$. It was possible that HPVs utilized this strategy for infection that initial binding to a primary receptor and then transfer to a secondary receptor allowing for invasion of cells. Thus, it was most likely that both $\alpha 6$ integrin and HSPGs, functioning as primary or secondary receptor, contributed individually or in combination to the process. It should be noted that a virus receptor means a host surface component involved in binding and facilitating a viral infection. Therefore, we believe that both $\alpha 6$ integrin and HSPGs can be labeled HPV receptor, and that more receptors will be identified in the future.

\section{HPVs to enter cells via distinct pathways}

Intriguingly, most of the studies proved that different types of HPVs entered cells in distinct pathways, including clathrin-mediated endocytosis, caveolar endocytosis, and clathrin- and caveolae-independent endocytosis.

\subsection{Clathrin-mediated endocytosis}

It was found that clathrin-mediated endocytosis was the major cellular entry for many viruses. The binding of ligand to a specific receptor is widely recognized to result in the clustering of the ligand-receptor complexes in the coated pits on the plasma membrane (Fig. 1A), which then invaginated and pinched off from the plasma membrane to form intracellular clathrin-coated vesicles in progress to early endosomes in a Rab5- dependent manner before being fused with each other to form late endosome or lysosome, controlled by Rab7. And the molecules internalized via clathrin-mediated endocytosis experience a fast decline in $\mathrm{pH}$ from the neutral to a $\mathrm{pH}$ approximately 6 in the early endosomes transforming into the late endosomes and ultimately degrading in lysosomes, with a $\mathrm{pH}$ of approximately $5[31,32]$.

Day et al examined the pathway via which papillomaviruses infected cells using BPV virions and VLPs, and concluded that the pathway was accomplished via clathrin-dependent receptor-mediated endocytosis, and the viral capsid, unlike other molecules, presented a conformational change when the $\mathrm{pH}$ of the endosomal compartment decreased, resulting in the endosomal escape of the viral genome or genome/L2 complex (Fig. 1B) [33]. Moreover, Bousarghin et al revealed that HPV-16, -31 , and -58 , which were closely related viruses, however, presented different endocytosis pathways, HPV-16 and -58 typically internalized through clathrin-coated vesicles, and HPV-31 most likely to involve caveolae [34]. However, one investigation reached a conclusion that HPV-31, as in the case of HPV-16, entered the human and primate cells through a clathrin-mediated pathway [35]. All suggests much ambiguity in terms of papillomavirus entry, which merits further studies.

\subsection{Caveolar endocytosis}

The number of viruses that enter cells via caveolar endocytosis as an alternative uptake pathway was found to be less than via clathrin-mediated endocytosis. In the former, most of these viruses were nonenveloped and less than $55 \mathrm{~nm}$ [31], as in the case of HPVs. Compared with the clathrin-mediated entry, caveolae performed internalization at a lower speed, the resulting vesicles failing to become acidized, and an additional difference was that internalization via caveolae was not a constitutive process [32]. Other studies showed that the caveolar endocytosis passed through the caveosomes, bypassing endosomes, and then moved to the Golgi body, and/or endoplasmic reticulum (Fig. 1C) [36], and HPV-31 was found to do this via caveolar endocytosis [34,36,37].

For years, clathrin-dependent endocytosis and caveolar entry were believed to be of two parallel but separate pathways. However, as indicated by the latest investigations, there was cross talk whereby cargo could move between them with some molecules involved. Rab 5 GTPase was first identified. Laniosz et al found that BPV-1 although shown to possess the entry capacity via clathrin-dependent endocytosis, was incapable of establishing an infection without caveolin-1, suggesting that the virus whose entry was facilitated via clathrinmediated endocytosis, utilized the caveolar pathway postentry for infection, where the Rab 5 might induce or be involved in its transport from the endosome to the caveosome (Fig. 1D) [38]. Afterwards, Smith et al reported that HPV-31, upon initial cellular binding and associating with caveolin-1, was transferred to the early endosome and proceeded through the endosomal pathway, during which the Rab 5 might be responsible for 


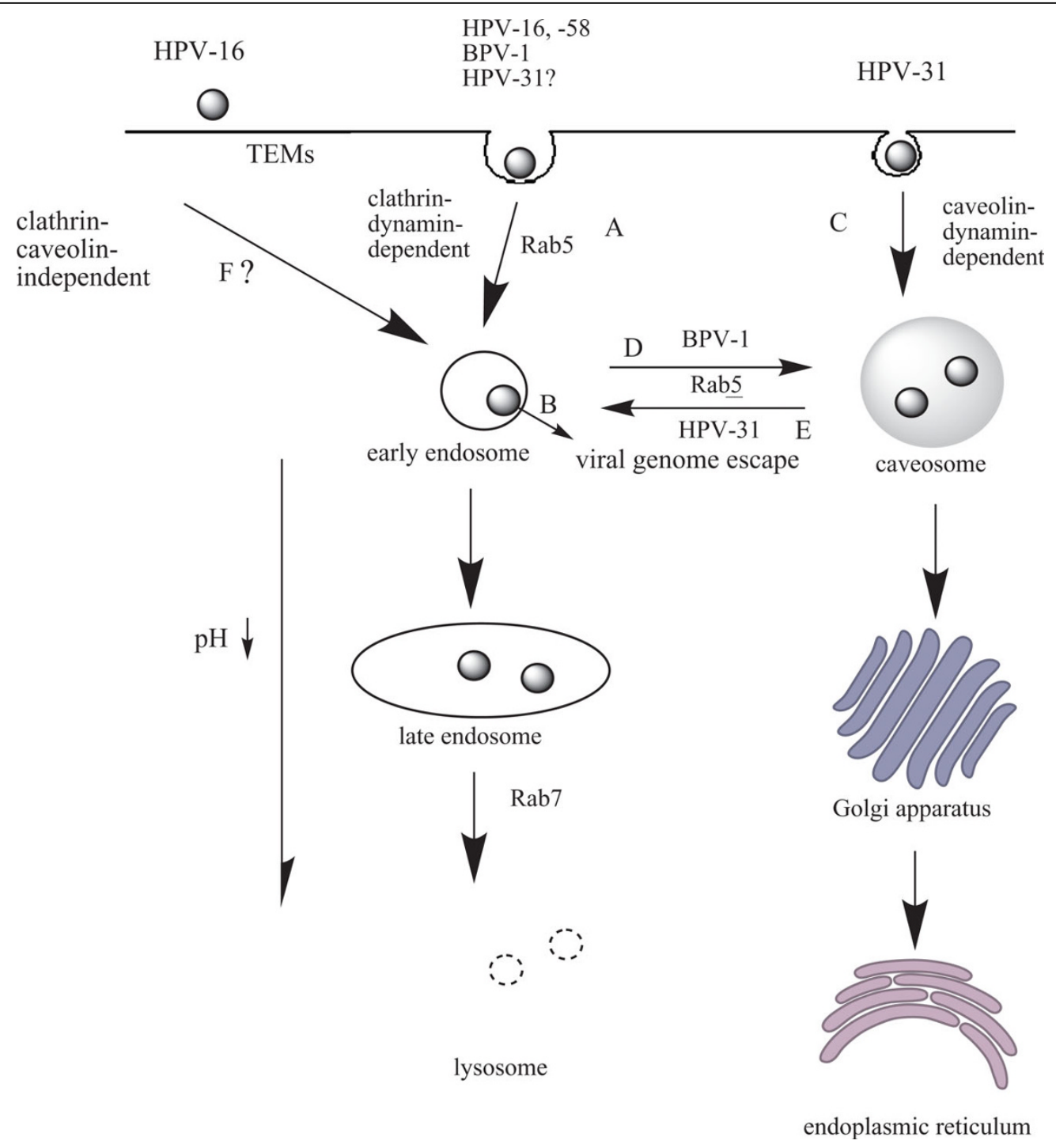

Figure 1 Endocytic pathways of HPVs. (A) HPV-16, -58, and BPV-1 entered via clathrin/dynamin dependent pathway. HPV-31 might enter via this pathway. (B) The decrease of $\mathrm{pH}$ led to endosomal escape of the viral genome. (C) HPV-31 entered via caveolin/dynamin dependent pathway. (D) and (E) Virions were transported between early endosome and caveosome. (F) HPV-16 might be internalized in clathrin- and caveolae-independent pathway, via TEMs.

the exchange of the cargo [38]. Another putative molecule might be dynamin, also called a GTPase, which was capable of affecting "pinching off" coated vesicles to form nascent clathrin-coated or caveolin-1-coated endocytic vesicles at the plasma membrane. It was reported that a dynamin inhibitor, dynasore, blocked the infection of HPV-16 and BPV-1 pseudovirions in a dose- and time-dependent manner with equal efficiency [39], and that HPV-31 infection could be blocked using a dynamin-2 dominant negative molecule [35].

\subsection{A Clathrin- and caveolae-independent pathway}

In a latest study, HPV-16 was reported to be capable of entering and thus infecting cells in a clathrin- and caveolae-independent manner, and further evidence indicated that tetraspanin-enriched microdomains (TEMs) were involved in the endocytosis (Fig. 1F) [40]. Tetraspanins are an evolutionary conserved family of four transmembrane domain-containing proteins including at least 32 members in humans [41], which are able to interact laterally with each other and with other transmembrane proteins to form TEMs, within which tetraspanins can control and modulate complicated activities including adhesion, migration, and synapse formation, as well as endocytosis and exocytosis [42]. Spoden et al proposed that HPV-16 particles, following binding to the cells, colocalized with the tetraspanins 
CD63 and CD151 whose capacity to interact with other membrane components and assemble into microdomains on the plasma membrane enabled these molecules to serve as the recipients of virions from the primary receptors, such as HSPGs. Consequently, the binding could trigger endocytic uptake processes and infection [40].

\section{L2 protein necessary for infection}

The function of L2 has long been neglected. Recently, a growing body of evidence has suggested that L2 is necessary for the establishment of HPV infection. L2 of all sequenced HPVs contain at their $\mathrm{N}$ termini a consensus cleavage motif for furin, a proprotein convertase, and furin cleavage is supposed to be necessary for cellular attachment and entry. Therefore, a model of L2 functioning in the early events of PV infection was proposed, in which the initial attachment to HSPGs moieties functioned primarily as the critical step of L2 cleavage by furin, thus resulting in a conformational change of viral capsids, followed by the capsids detaching from HSPGs and associating with a putative second receptor [43]. Other studies showed that furin cleavage might occur at the cell surface or within an early endosomal compartment [44], and the capsids underwent uncoating in a late endosomal compartment, leading to the associated genome to escape from the endosome into the cytoplasm via a mechanism that involved the C-terminus of L2 [45].

In summary, HPVs had to undergo a complicated process to successfully infect their host cells. We presented cellular receptor-binding and internalization pathways of HPVs, which were of multiple steps relating to numerous molecules, cellular or viral, suggesting that it was a promising step in attacking pathogenic viruses before they could utilize the host cell's machinery for replication, and the studies on HPV cellular binding and entry would locate novel molecular targets for antiviral strategies.

\section{Acknowledgements}

We would like to thank Dr. Xie, Youzhou and Fu, Yaoyao for their helpful technical instructions. And we feel grateful to all the authors for their citations in the review.

\section{Author details}

${ }^{1}$ Department of Otolaryngology, Eye and ENT Hospital of Fudan University (formerly Shanghai Medical University), Shanghai, China. ${ }^{2}$ Department of Otolaryngology, Eye and ENT Hospital, Fudan University, Shanghai, China.

Authors' contributions

Both authors contributed to the original drafts of the manuscript, and approved the final version.

\section{Competing interests}

The authors declare that they have no competing interests.
Received: 11 August 2009

Accepted: 6 January 2010 Published: 6 January 2010

\section{References}

1. Roden RB, Kirnbauer R, Jenson AB, Lowy DR, Schiller JT: Interaction of papillomaviruses with the cell surface. J Virol 1994, 68:7260-7266.

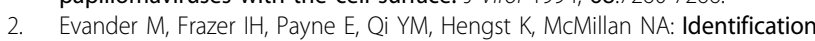
of the alpha6 integrin as a candidate receptor for papillomaviruses. $J$ Virol 1997, 71:2449-2456.

3. Patterson NA, Smith $J$, Ozbun MA: Human papillomavirus type $31 \mathrm{~b}$ infection of human keratinocytes does not require heparan sulfate. $J$ Virol 2005, 79:6838-6847.

4. Conway MJ, Meyers C: Replication and assembly of humanpapillomaviruses. J Dent Res 2009, 88:307-317.

5. Wang HK, Duffy AA, Broker TR, Chow LT: Robust production and passaging of infectious HPV in squamous epithelium of primary human keratinocytes. Genes Dev 2009, 23:181-194.

6. Chow LT, Duffy AA, Wang HK, Broker TR: A highly efficient system to produce infectious human papillomavirus: Elucidation of natural virushost interactions. Cell Cycle 2009, 8:1319-1323.

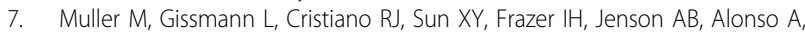
Zentgraf $H$, Zhou J: Papillomavirus capsid binding and uptake by cells from different tissues and species. J Virol 1995, 69:948-954.

8. Volpers C, Unckell F, Schirmacher P, Streeck RE, Sapp M: Binding and internalization of human papillomavirus type 33 virus-like particles by eukaryotic cells. J Virol 1995, 69:3258-3264.

9. Qi YM, Peng SW, Hengst K, Evander M, Park DS, Zhou J, Frazer $\mathrm{H}_{\text {: Epithelial }}$ cells display separate receptors for papillomavirus VLPs and for soluble L1 capsid protein. Virology 1996, 216:35-45.

10. McMillan NA, Payne E, Frazer $\mathbb{H}$, Evander M: Expression of the alpha6 integrin confers papillomavirus binding upon receptor-negative B-cells. Virology 1999, 261:271-279

11. Yoon CS, Kim KD, Park SN, Cheong SW: alpha(6) Integrin is the main receptor of human papillomavirus type 16 VLP. Biochem Biophys Res Commun 2001, 283:668-673.

12. Giancotti FG, Stepp MA, Suzuki S, Engvall E, Ruoslahti E: Proteolytic processing of endogenous and recombinant beta 4 integrin subunit. $J$ Cell Biol 1992, 118:951-959.

13. Kajiji S, Tamura RN, Quaranta V: A novel integrin (alpha E beta 4) from human epithelial cells suggests a fourth family of integrin adhesion receptors. EMBO J 1989, 8:673-680.

14. Kurpakus MA, Quaranta V, Jones JC: Surface relocation of alpha 6 beta 4 integrins and assembly of hemidesmosomes in an in vitro model of wound healing. J Cell Biol 1991, 115:1737-1750.

15. Bretscher MS: Cells can use their transferrin receptors for locomotion. EMBO J 1992, 11:383-389.

16. Sonnenberg A, Calafat J, Janssen $H$, Daams $H$, Raaij-Helmer van der LM, Falcioni R, Kennel SJ, Aplin JD, Baker J, Loizidou M, Garrod D: Integrin alpha $6 /$ beta 4 complex is located in hemidesmosomes, suggesting a major role in epidermal cell-basement membrane adhesion. J Cell Biol 1991, 113:907-917.

17. Selinka HC, Giroglou T, Nowak T, Christensen ND, Sapp M: Further evidence that papillomavirus capsids exist in two distinct conformations. J Virol 2003, 77:12961-12967.

18. Knappe M, Bodevin S, Selinka HC, Spillmann D, Streeck RE, Chen XS, Lindahl U, Sapp M: Surface-exposed amino acid residues of HPV16 L1 protein mediating interaction with cell surface heparan sulfate. J Biol Chem 2007, 282:27913-27922

19. Joyce JG, Tung JS, Przysiecki CT, Cook JC, Lehman ED, Sands JA, Jansen KU, Keller PM: The L1 major capsid protein of human papillomavirus type 11 recombinant virus-like particles interacts with heparin and cell-surface glycosaminoglycans on human keratinocytes. J Biol Chem 1999, 274:58105822

20. Giroglou T, Florin L, Schafer F, Streeck RE, Sapp M: Human papillomavirus infection requires cell surface heparan sulfate. J Virol 2001, 75:1565-1570

21. Combita AL, Touze A, Bousarghin L, Sizaret PY, Munoz N, Coursaget P: Gene transfer using human papillomavirus pseudovirions varies according to virus genotype and requires cell surface heparan sulfate. FEMS Microbiol Lett 2001, 204:183-188. 
22. Selinka HC, Giroglou T, Sapp M: Analysis of the infectious entry pathway of human papillomavirus type 33 pseudovirions. Virology 2002, 299:279287.

23. Shafti-Keramat S, Handisurya A, Kriehuber E, Meneguzzi G, Slupetzky K, Kirnbauer R: Different heparan sulfate proteoglycans serve as cellular receptors for human papillomaviruses. J Virol 2003, 77:13125-13135.

24. Rommel O, Dillner J, Fligge C, Bergsdorf C, Wang X, Selinka HC, Sapp M: Heparan sulfate proteoglycans interact exclusively with conformationally intact HPV L1 assemblies: basis for a virus-like particle ELISA. J Med Virol 2005, 75:114-121.

25. Chen XS, Garcea RL, Goldberg I, Casini G, Harrison SC: Structure of small virus-like particles assembled from the $\mathrm{L} 1$ protein of human papillomavirus 16. Mol Cell 2000, 5:557-567.

26. Modis $Y$, Trus BL, Harrison SC: Atomic model of the papillomavirus capsid. EMBO J 2002, 21:4754-4762.

27. Day PM, Thompson CD, Buck CB, Pang YY, Lowy DR, Schiller JT: Neutralization of human papillomavirus with monoclonal antibodies reveals different mechanisms of inhibition. J Virol 2007, 81:8784-8792.

28. Carter JJ, Wipf GC, Benki SF, Christensen ND, Galloway DA: Identification of a human papillomavirus type 16-specific epitope on the C-terminal arm of the major capsid protein L1. J Virol 2003, 77:11625-11632.

29. Sibbet G, Romero-Graillet C, Meneguzzi G, Campo MS: alpha6 integrin is not the obligatory cell receptor for bovine papillomavirus type 4. J Gen Virol 2000, 81:327-334.

30. Johnson KM, Kines RC, Roberts JN, Lowy DR, Schiller JT, Day PM: Role of heparan sulfate in attachment to and infection of the murine female genital tract by human papillomavirus. J Virol 2009, 83:2067-2074.

31. Mudhakir D, Harashima $\mathrm{H}$ : Learning from the viral journey: how to enter cells and how to overcome intracellular barriers to reach the nucleus. AAPS J 2009, 11:65-77.

32. Sieczkarski SB, Whittaker GR: Dissecting virus entry via endocytosis. J Gen Virol 2002, 83:1535-1545.

33. Day PM, Lowy DR, Schiller JT: Papillomaviruses infect cells via a clathrindependent pathway. Virology 2003, 307:1-11.

34. Bousarghin $L$, Touze $A$, Sizaret $P Y$, Coursaget $P$ : Human papillomavirus types 16, 31, and 58 use different endocytosis pathways to enter cells. J Virol 2003, 77:3846-3850.

35. Hindmarsh PL, Laimins LA: Mechanisms regulating expression of the HPV 31 L1 and L2 capsid proteins and pseudovirion entry. Virol J 2007, 4:19.

36. Smith JL, Campos SK, Ozbun MA: Human papillomavirus type 31 uses a caveolin 1- and dynamin 2-mediated entry pathway for infection of human keratinocytes. J Virol 2007, 81:9922-9931, Laniosz V, Holthusen KA Meneses PI: Bovine papillomavirus type 1: from clathrin to caveolin. J Virol 2008, 82:6288-6298..

37. Smith JL, Campos SK, Wandinger-Ness A, Ozbun MA: Caveolin-1dependent infectious entry of human papillomavirus type 31 in human keratinocytes proceeds to the endosomal pathway for $\mathrm{pH}$-dependent uncoating. J Virol 2008, 82:9505-9512.

38. Laniosz V, Holthusen KA, Meneses PI: Bovine papillomavirus type 1: from clathrin to caveolin. J Virol 2008, 82:6288-6298.

39. Abban CY, Bradbury NA, Meneses PI: HPV16 and BPV1 infection can be blocked by the dynamin inhibitor dynasore. Am J Ther 2008, 15:304-311.

40. Spoden G, Freitag K, Husmann M, Boller K, Sapp M, Lambert C, Florin L: Clathrin- and caveolin-independent entry of human papillomavirus type 16-involvement of tetraspanin-enriched microdomains (TEMs). PLoS One 2008, 3:e3313.

41. Hemler ME: Tetraspanin proteins mediate cellular penetration, invasion, and fusion events and define a novel type of membrane microdomain. Annu Rev Cell Dev Biol 2003, 19:397-422.

42. Levy S, Shoham T: Protein-protein interactions in the tetraspanin web. Physiology (Bethesda) 2005, 20:218-224.

43. Day PM, Lowy DR, Schiller JT: Heparan sulfate-independent cell binding and infection with furin-precleaved papillomavirus capsids. J Virol 2008, 82:12565-12568.

44. Richards RM, Lowy DR, Schiller JT, Day PM: Cleavage of the papillomavirus minor capsid protein, L2, at a furin consensus site is necessary for infection. Proc Natl Acad Sci USA 2006, 103:1522-1527.

45. Kamper N, Day PM, Nowak T, Selinka HC, Florin L, Bolscher J, Hilbig L, Schiller JT, Sapp M: A membrane-destabilizing peptide in capsid protein L2 is required for egress of papillomavirus genomes from endosomes. J Virol 2006, 80:759-768.
doi:10.1186/1743-422X-7-2

Cite this article as: Letian and Tianyu: Cellular receptor binding and entry of human papillomavirus. Virology Journal 2010 7:2.

\section{Publish with Bio Med Central and every scientist can read your work free of charge}

"BioMed Central will be the most significant development for disseminating the results of biomedical research in our lifetime. "

Sir Paul Nurse, Cancer Research UK

Your research papers will be:

- available free of charge to the entire biomedical community

- peer reviewed and published immediately upon acceptance

- cited in PubMed and archived on PubMed Central

- yours - you keep the copyright
BioMedcentral 